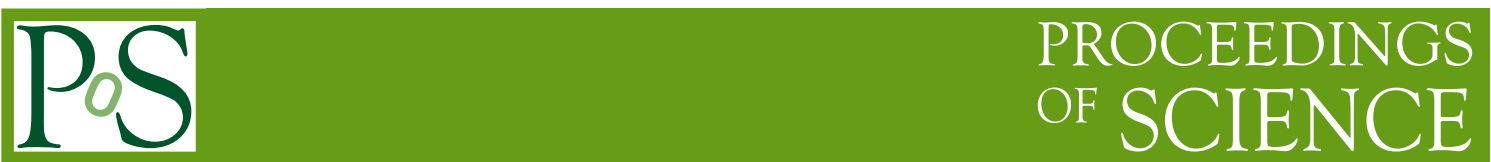

\title{
OH MM activity and the ULIRG - SMG source population
}

\section{Willem A. Baan*}

ASTRON, Dwingeloo, The Netherlands

E-mail: baaneastron.nl

\section{Hans-Rainer Klöckner}

University of Oxford, United Kingdom

E-mail: hrkeastro.ox.ac.uk

\begin{abstract}
$\mathrm{OH}$ Megamaser (OH MM) galaxies are part of the nearby population of Luminous Infrared Galaxies. Under the assumption that $\mathrm{OH}$ Megamaser activity will also follow the much larger population of Sub-Millimetre Galaxies, predictions can be made of the number of detectable $\mathrm{OH}$ MMs versus redshift. Known luminosity functions of the FIR and $\mathrm{OH}$ MM source populations have been evaluated by introducing various forms of number evolution for the FIR source population. In addition, number evolution has been considered for the relative fraction of high-luminosity $\mathrm{OH}$ MMs among the FIR source population. The predictions show that without any FIR number evolution $\mathrm{OH} \mathrm{MM}$ can be found only in the nearby universe. With number evolution $\mathrm{OH} M \mathrm{MM}$ may be detected to redshifts of $4-5$. The results also indicate that $\mathrm{OH}$ MM may be used to constrain the galaxy merger rates and the number evolution of the ULIRG/SMG population.
\end{abstract}

Panoramic Radio Astronomy: Wide-field 1-2 GHz research on galaxy evolution - PRA2009

June 02 - 052009

Groningen, the Netherlands

${ }^{*}$ Speaker. 


\section{Introduction}

Ultra-Luminous Infrared Galaxies (ULIRGs) represent a short-lived (about 1 Gyr) phenomenon in the life of galaxies resulting from episodes of mass assembly and mostly star-formation driven nuclear activity (Vernet et al. 2001). Evidence is mounting that sub-millimetre galaxies (SMGs) are the high-redshift versions of nearby ULIRGs and are more numerous showing a peak in number density at $z=2-4$ (see Aretxaga et al. 2007). Most SMGs are detected in deep radio surveys up to $z \approx 5.2$ (at 1.4GHz; Chapman et al. 2005) and they follow the well-known FIR-radio correlation.

The SHADES survey of the high-redhsift source population using the SCUBA instrument at JCMT has been sensitive to SMGs in the range $L_{\mathrm{FIR}}=2.6 \times 10^{12}-10^{13} \mathrm{~L}_{\odot}$ (Aretxaga et al. 2007; Serjeant et al. 2008). The similar AZTEC survey using the Mambo instrument at the IRAM 30m Pico Veleta telescope covered the range $L_{\mathrm{FIR}}=10^{11}-10^{12} \mathrm{~L}_{\odot}$ (Scott et al. 2008). These studies reveal the star-formation rate and SF energy density as function of redshift and show that starformation accounts for minimally $65 \%$ of the energy generation with the rest provided by AGNs.

Known OH MegaMasers (MM) and Gigamasers (GM) are found in the nearby ULIRG population with $L_{\text {FIR }}$ up to $10^{13} \mathrm{~L}_{\odot}$ and would also follow the high-z ULIRG/SMG population. So far, the directed (nearby) $\mathrm{OH}$ searches have been limited by the availability of IRAS redshifts (up to $z$ $=0.26$ ) and there is a need for blind (and non-blind) searches for OH MM.

In this paper, we consider predictions of the detection rate and number densities of $\mathrm{OH} \mathrm{MMs}$ as a function of redshift for sensitivity limited surveys. In order to increase the detection rate, searches would benefit from studies that concentrate on redshift determinations, radio correlations, and population modeling of the SMG population. Blind searches would coincide with HI detection survey above $1000 \mathrm{MHz}(z(H I)=0.4)$. Such studies will elucidate evolutionary differences between SMGs and ULIRG populations and highlight the number evolution of the FIR population.

\section{OH MegaMaser properties and luminosity functions}

An OH MM luminosity function based on the Arecibo sample of 53 sources (Darling \& Giovanelli 2002a) has been presented as a power law from $\log L_{\mathrm{OH}}=2-3.8$ using a Malmquist correction to correct for weaker sources that could be missing in surveys. An FIR luminosity function of $\mathrm{OH}$ MegaMasers has been obtained by Klöckner (2004) for a sample of 73 reliable sources (Figure 1) using Schechter functions for the LFs for OH MMs and FIR sources. The properties shape of this OH MM LF is in general agreement with the LF presented earlier (Baan 1991, 1997) except that the fraction of $\mathrm{OH}$ MMs among the FIR population is somewhat smaller.

The OH MegaMaser sources comprise a variable fraction $F_{O H-F I R}$ of the ULIRG population with a maximum of $5 \%$ in the luminosity range of $\log L_{\mathrm{FIR}}=10.6-11.5 \mathrm{~L}_{\odot}$ and decreasing at both ends (Figure 1; Baan 1997; Klöckner 2004). This fraction may be explained by the opening angle of the amplifying medium or the duty cycle of MM activity during FIR outburst (a rough estimate of $10 \%$ ). Alternatively, the variation of $F_{O H-F I R}$ with $L_{\mathrm{FIR}}$ could result from sample incompleteness or variation in the pumping efficiency. A $V / V_{m}$ test shows that the sample is incomplete outside the $10^{2.1} \mathrm{~L}_{\odot}<L_{\mathrm{OH}}<10^{3} \mathrm{~L}_{\odot}$ range (Klöckner 2004).

The $L_{\mathrm{OH}}$ varies non-linearly with $L_{\mathrm{FIR}}$ as would be expected for a (simple) continuum amplification scenario. Assuming $L_{\mathrm{OH}} \propto L_{\mathrm{FIR}}{ }^{\alpha}$, early observations were found to be consistent with 
the predicted slope of $\alpha=2$ (see Baan 1989). Klöckner (2004) finds $\alpha=2.29$ for a sample of 73 reliable sources. On the other hand, Darling \& Giovanelli (2002b) find a smaller slope of $\alpha=1.57$ after consideration of the Malmquist bias correction. However, for a peak flux limited sample of amplified maser emission the pumping mechanism is an important factor and it is unlikely that the Malmquist bias correction is the right approach.

A first estimate of the number of detectable $\mathrm{OH} \mathrm{MM}$ has been presented by Briggs (1998). These estimates were based on early $\mathrm{OH}$ MM LFs, using a quadratic $L_{\mathrm{OH}}-L_{\mathrm{FIR}}$ relation, and an optimistic $F_{O H-F I R}$ fractions (Baan 1991, 1997). The variation of the galaxy merger rate with redshift was used as an indicator of the number evolution (NE) of FIR sources. This study also confirms that the observable population of HI sources would extend to $z=0.4$.

The number of observable $\mathrm{OH} \mathrm{MM}$ may be determined by considering the $\mathrm{OH}$ MM population as a subset of the known FIR population or as a standalone population. In this paper we employ the FIR LF of $\mathrm{OH} \mathrm{MM}$ as presented by Klöckner (2004) and using the LF of OH MM as a parametrized fraction of the LF of FIR sources (Figure 1). Taking the number and/or luminosity evolution for the FIR and OH MM LFs into account, the number of detectable $\mathrm{OH}$ sources can then be determined as a function of redshift and observational sensitivity.

\section{Redshift evolution of the FIR and OH MM populations}

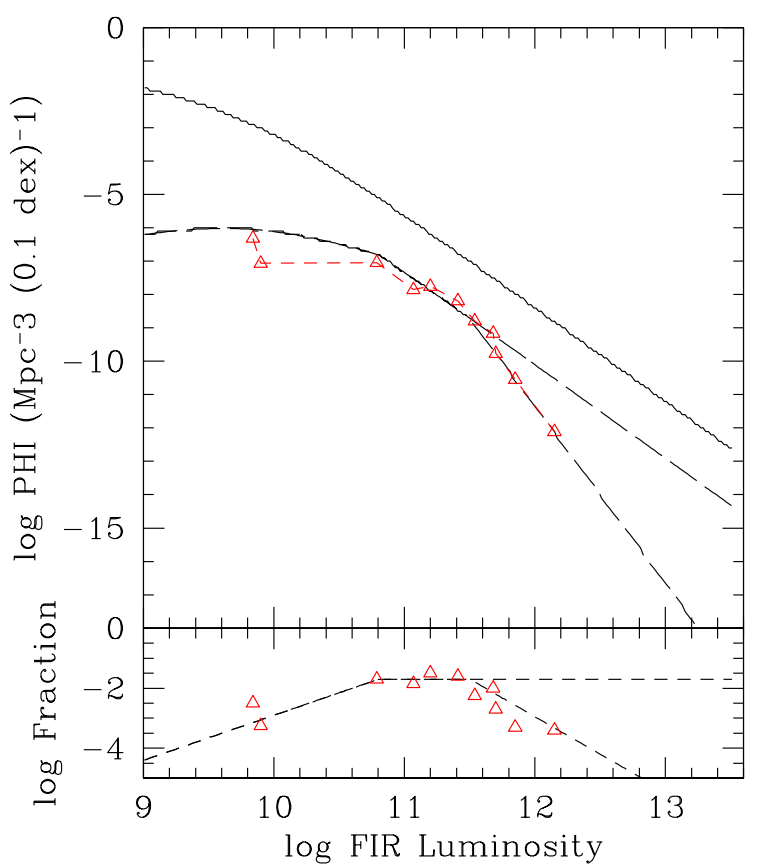

Figure 1: $\mathrm{OH} M M$ and FIR luminosity functions. The FIR Luminosity is based on the $60 \mu \mathrm{m}$ luminosity. Upper Frame: The solid line is a Schechter function representing the FIR galaxy population. The open triangles represent the $\mathrm{OH} \mathrm{MM}$ population with two dashed curves are Schechter approximations relating to two approximations presented in the lower frame. Lower Frame: The observed fraction $F_{O H-F I R}$ has been depicted with the open triangles. The dashed lines represent two parameterizations of the observed OH MM population. The flat curve depicts luminosity/number evolution at higher luminosities. Data obtained from Klöckner 2004.

The variation of star-formation rate with redshift may provide a reliable estimate of the number or luminosity evolution (NE and LE) of the FIR source population in the observable universe. The galaxy merger rate is known to sharply increase with redshift and provides a causal relation between star-formation, FIR prominence and OH MM activity. Briggs (1998) adopted $N E(z)=(1+z)^{n}$ to estimate the enhancement in the merger rate with $n=0$ for no number evolution, $\mathrm{n}=4$ or 8 until $\mathrm{z}=2.5$ and constant beyond 2.5 for moderate and strong evolution $(N E(z)$ enhancement factors are respectively 1,81 , and 6561 at $z=2$ ). Based on the data presented by Aretxaga et al. (2007) and others, a parabolic approximation of $N(z)$ may be considered to express the moderate number 

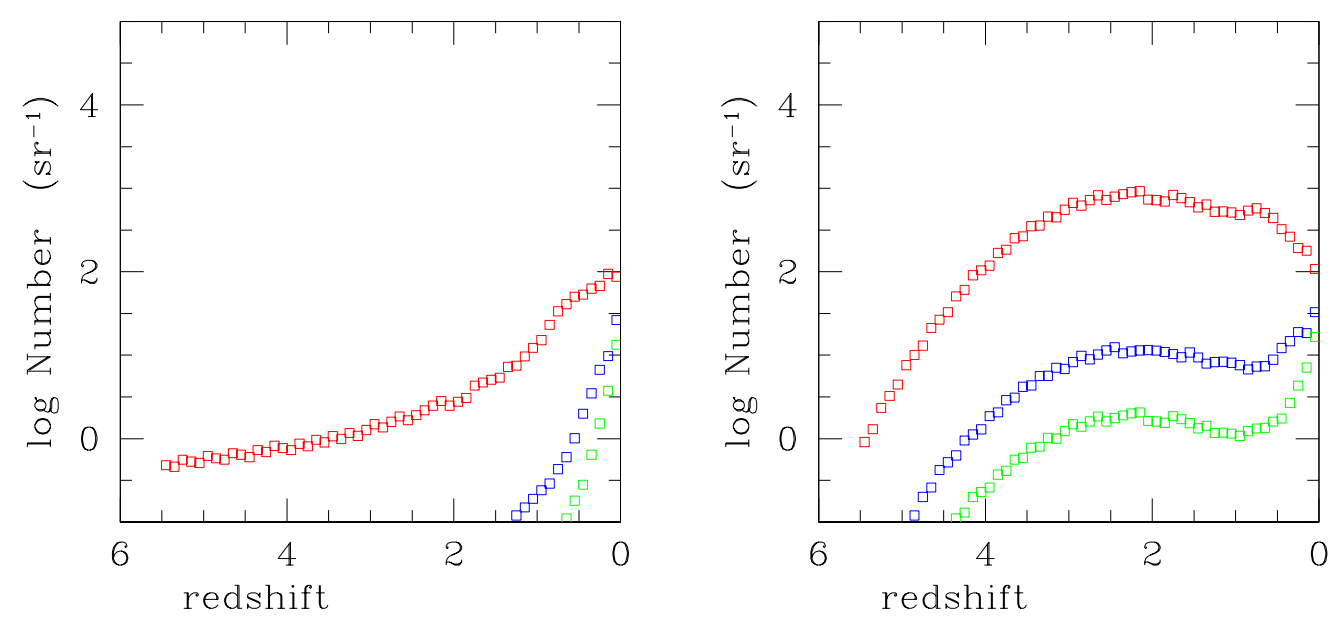

Figure 2: Prediction of $\mathrm{OH} M \mathrm{MM}$ detections for the known $\mathrm{OH} \mathrm{LF}$ and considering FIR number evolution. A three-component fraction has been used with decreasing $F_{O H-F I R}$ at both low and high $\mathrm{OH}$ luminosities as depicted in Figure 1. The left frame assumes no FIR number evolution. The right frame assumes a parabolic FIR number evolution as described in section 3. The curves are for sensivities of $20 \mathrm{mJy}$ (upper curve; red), $2 \mathrm{mJy}$ (middle curve; blue), and $0.2 \mathrm{mJy}$ (lower curve; green) per $100 \mathrm{~km} / \mathrm{s}$ channel width. The redshift is binned in units of 0.1 .

evolution of SMG that peaks at $z=2.8: \rho_{S F}=457 \exp \left[-0.5(z-2.8)^{2} / 0.8^{2}\right]$. In this paper, we only consider the two NE cases for the FIR population: no evolution and moderate parabolic evolution.

The LF of OH MM has some uncertainty at the high-luminosity end and Fig. 1 presents a alternative parametrization of the fraction $F_{O H-F I R}$ that incorporates number (or luminosity) evolution. Enhancing the fraction at the high- $L_{\mathrm{FIR}}$ end would be consistent with our understanding of the $\mathrm{OH} \mathrm{MM}$ phenomenon and would address the completeness in this range. In this regard, we consider two scenarios as depicted in Fig. 1, as follows: (a) the fraction $F_{O H-F I R}$ with decreasing sections at low and high FIR luminosities (Klöckner 2004), and (b) the $F_{O H-F I R}$ as a constant until $L_{\mathrm{FIR}}=10^{13.5} \mathrm{~L} \odot_{\odot}$.

\section{Predictions for detections of $\mathrm{OH}$ MM/GM}

The characteristics of the $\mathrm{OH}$ MM population have been used to predict the number of observable sources as a function of redshift. For this purpose we assume that the velocity width of the strongest $1667 \mathrm{MHz} \mathrm{OH}$ emission component is contained within a $100 \mathrm{~km} / \mathrm{s}$ spectral channel and that the detection levels are at $0.2,2$ and $20 \mathrm{mJy} / \mathrm{channel}$. Predictions for detectable $\mathrm{OH} \mathrm{MM}$ considering the FIR number evolution are presented in both Figs. 2 and 3, while the modified predictions for $\mathrm{OH} \mathrm{MM}$ number evolution are presented in Fig. 3.

The detection of $\mathrm{OH}$ MM depends strongly on the emission line profiles of the sources. Because of the amplification process and the intrinsic line ratio of the 1667 and $1665 \mathrm{MHz}$ line pair, the powerful sources have a $1667 \mathrm{MHz}$ component that is much stronger than the $1665 \mathrm{MHz}$. The line-width of the $1667 \mathrm{MHz}$ mostly depends on the size and dynamics of the nuclear region and may vary between 50 to $300 \mathrm{~km} / \mathrm{s}$. However, the two (most distant) OH GigaMaser sources have very 

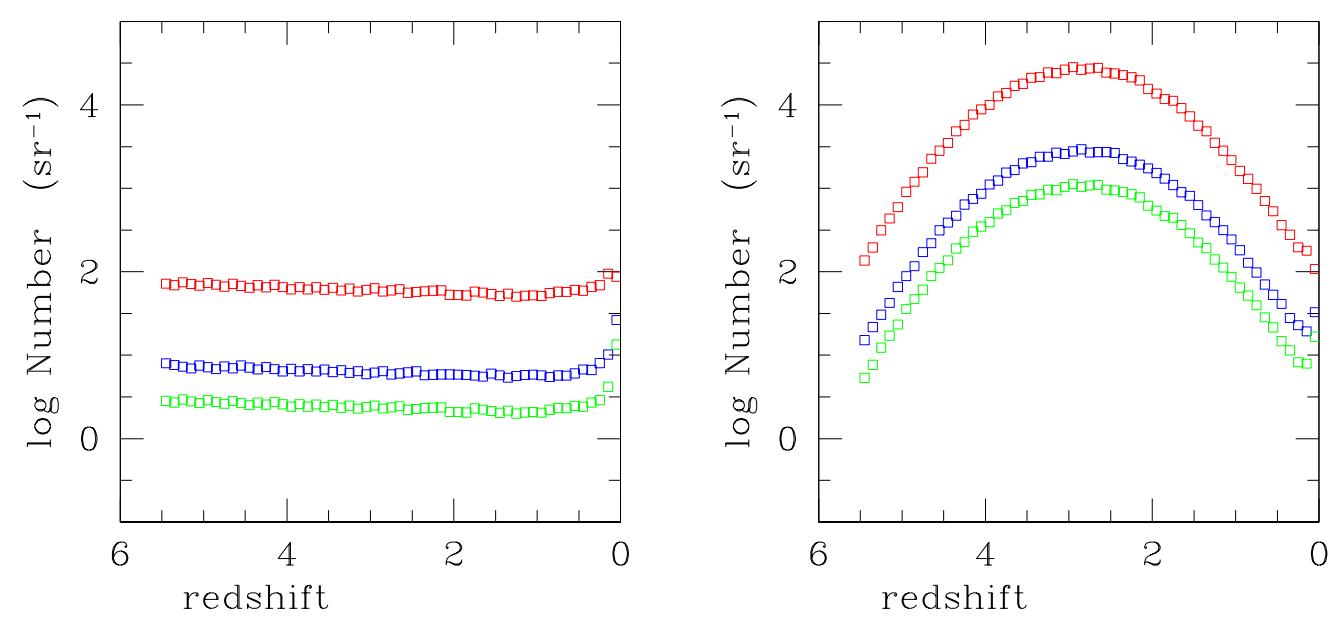

Figure 3: Prediction of $\mathrm{OH} M M$ detections for $\mathrm{NE}$ for $\mathrm{OH} M \mathrm{MM}$ and also considering FIR number evolution. A two-component representation $F_{O H-F I R}$ has been used that decreases at low $\mathrm{OH}$ luminosities and has a constant fraction at high luminosities as depicted in Figure 1. The left frame assumes no FIR number evolution. The right frame assumes a parabolic FIR number evolutions as described in section 3. For further explanation see Figure 2.

broad multi-component emission profiles that cover more than $2000 \mathrm{~km} / \mathrm{s}$. A broadened OH GM profiles limits the maximum redshift $z(\max )$ for detection, which would require an even coarser spectral resolution. Similarly, the variation of $L_{\mathrm{OH}}$ for each $L_{\mathrm{FIR}}$ also modifies the detection probability. HI contamination in blind surveys at frequencies above $1000 \mathrm{MHz}$ requires differentiation of emission profiles of $\mathrm{OH}$ and $\mathrm{HI}$ sources with the help of optical redshift determinations.

The detection of OH GM at high redshift depends on the existence of a population of highluminosity FIR sources, any luminosity evolution for the OH MM population, and the maximum possible $L_{\mathrm{OH}}$. Known Gigamasers with $L_{\mathrm{OH}}=10^{4.15} \mathrm{~L}_{\odot}$ could be seen up to $z=3$ while sources with $10^{4.5} \mathrm{~L}_{\odot}$ could be seen up to $z=4$. The actual maximum value for $L_{\mathrm{OH}}$ would be limited by (a) a lower pumping efficiency at high $L_{\mathrm{FIR}}$, (b) variation of abundance of $\mathrm{OH}$ molecules in the gas phase of the ISM during the early stages of nuclear evolution, and (c) changes in the duty cycle of $\mathrm{OH} \mathrm{MM}$ activity during nuclear evolution.

\section{Determining the Luminosity Functions of the FIR and OH MM populations}

The diagrams of Figure 2 and 3 show that the currently know OH MM population may be detected up till $z=4$ at the highest sensitivity even without any number evolution. Without any number evolution $\mathrm{OH} \mathrm{MM}$ can be found only in the nearby universe. For parabolic number evolution, the $\mathrm{OH} \mathrm{MM}$ population would follow the FIR population with significant numbers to be found up to $z=5$ (Fig. 2 right). For OH luminosity evolution, the detectable $\mathrm{OH}$ population follows the highest luminosity FIR population. The differentiation between models at $z<1$ may be readily used to evaluate the galaxy merger rates and the number or luminosity evolution of the ULIRG/SMG population. There remains an ambiguity about the number evolution in the $\mathrm{OH} M \mathrm{M}$ population, which may relate to number evolution of the FIR population or to the $\mathrm{OH}$ MM popula- 
tion by modifying the fraction. This distinction can be made when both LFs are determined using multiple redshift/frequency windows.

The differentiation in the detection rates with redshift may reveal the effects of number evolution of the FIR and $\mathrm{OH}$ population. The effect of luminosity/number evolution at high $\mathrm{OH} \mathrm{MM}$ luminosities may be seen at low redshifts up to unity even without changing the $\mathrm{OH} \mathrm{MM}$ fraction. The choice between shallow wide fields and deep narrow fields can thus be made after evaluating nearby searches. However, large scale surveys may be required to verify low detection rates.

\section{Summary ULIRGS and SMGs}

In the nearby universe, $\mathrm{OH}$ MM activity is found in a relatively small fraction of (U)LIRGs during fraction of the lifetime of the FIR/nuclear outburst. Therefore, OH MM activity provides diagnostics during the evolutionary sequence of (U)LIRGs-SMGs and the state of its nuclear ISM. The nature of ULIRGs-SMGs may suggest that the $\mathrm{OH}$ MM activity stage at higher redshift would become longer and more prominent. Furthermore $\mathrm{OH}$ MM activity complements the other surveys that study the SMG population and connect the radio continuum and IR properties.

Surveys for $\mathrm{OH} \mathrm{MM}$ can be done in parallel with $\mathrm{HI}$ and continuum surveys in spectral line mode. However, because of the masering process, the $\mathrm{OH}$ MM population extends further than the limits limits for detection of thermal HI emission. Surveys with current instruments such as the WSRT and GMRT and in the near future ASKAP and MeerKat will provide the initial information on the populations. Early directed searches have been mostly non-successful (and mostly unpublished). New surveys are underway. Theoretical modeling results presented here would suggest that the detectable $\mathrm{OH}$ MM population among the ULIRG/SMG population would be significant.

A final note is that RFI mitigation issues and spectrum management for existing and new telescope facilities are of great importance for sensitive surveys around $1 \mathrm{GHz}$ and below.

\section{References}

[1] Aretxaga, I. et al. 2007, MNRAS 379, 1571

[2] Baan, W.A. 1989, ApJ 338, 804

[3] Baan, W.A. 1991, in ASP 16 Atoms, Ions \& Molecules, eds A.D. Haschick \& P.T.P Ho, (ASP), 45

[4] Baan, W.A. 1997, in High Sensitivity Radio Astronomy, eds. N. Jackson \& R.J. Davis, (CUP), 73

[5] Briggs, F.H. 1991, A\&A 336, 815

[6] Chapman, S.C. et al. 2006, ApJ 622, 772

[7] Darling, J. \& Giovanelli, R. 2002a, ApJ 572, 810

[8] Darling, J. \& Giovanelli, R. 2002b, AJ 124, 100

[9] Klöckner, H.-R. 2004, PhD Thesis, University of Groningen

[10] Klöckner, H.-R. \& Baan, W.A. 2009, in preparation

[11] Serjeant, S. et al. 2008, MNRAS 386, 1907

[12] Scott, K.S. et al., 2008, MNRAS 385, 2225

[13] Vernet, J. et al. 2001, A\&A 366, 7 